\title{
«Transformation, innovation and adaptation for sustainability. Integrating natural and social sciences »
}

\author{
Compte rendu de conférence (Ljubljana, Slovénie, 29 juin-2 juillet 2009)
}

\author{
Markku Lehtonen \\ Économiste de l'environnement, Sussex Energy Group, SPRU - Science and Technology Policy Research, The Freeman Centre, \\ University of Sussex, Brighton BN1 9QE, Royaume-Uni
}

L'European Society for Ecological Economics (ESEE)1] avait choisi, pour la première fois depuis sa création en 1996, d'organiser sa $8^{\text {e }}$ conférence internationale dans un État d'Europe centrale et orientale. Plus de 250 participants venus de 33 pays différents ont participé à cette rencontre qui s'est déroulée à la Faculté de biotechnologie de 1'Université de Ljubljana? Le choix - notable - du pays d'accueil, ainsi que le nombre croissant de participants au fil des années, témoignent du dynamisme de ce courant de pensée économique hétérodoxe 3 .

\section{Quelques interventions marquantes}

Le thème de la conférence reflétait le désir, au sein de la communauté des économistes écologiques, de renforcer l'intégration entre les sciences sociales et les sciences naturelles. Bien que cet objectif de pluridisciplinarité soit, depuis le début, au cœur de l'économie écologique, l'implication des chercheurs en sciences naturelles reste, en

\footnotetext{
Auteur correspondant : M.Lehtonen@sussex.ac.uk

${ }^{1}$ En français, Société européenne pour l'économie écologique. $C f$. http://www.euroecolecon.org

${ }^{2}$ Le programme complet de cette conférence ainsi que le résumé et/ou le texte des communications sont disponibles sur le site Internet http://www.esee2009.si

3 L'économie écologique est un courant de pensée pluridisciplinaire (sciences économiques, sociales et naturelles), dédié à la recherche de nouvelles approches des questions de développement économique face à la montée des problèmes d'environnement et de développement durable. Contrairement à l'économie néoclassique dominante qui place l'économie au cœur de l'analyse, elle part du constat que toute activité humaine et économique est dépendante de l'écosphère qui l'entoure ( $c f$. http://www.euroecolecon.org/whatis.htm).
}

pratique, faible - l'économie écologique est, après tout, une science sociale.

La diversité des disciplines des conférenciers, qui sont intervenus aux séances d'ouverture et de clôture ainsi qu'aux sessions semi-plénières, est un autre signe de cette ambition de pluralisme. Lors de la séance d'ouverture, qui a porté sur l'importance et la pertinence de la théorie des jeux comportementale en politique sociale, Herbert Gintis (économiste, Santa Fe Institute, États-Unis) a trouvé le ton approprié, avec juste ce qu'il faut de provocation, pour lancer les débats : il a en effet attaqué quelques-uns des principes fondateurs de l'économie écologique, notamment l'hétérodoxie et le pluralisme méthodologique. «Laisser toutes les fleurs fleurir » reléguerait ce courant en marge et le rendrait impuissant face à l'école de pensée dominante et au monde politique. L'ambition de tout courant de pensée, selon H. Gintis, devrait être de gagner la bataille et non pas de se contenter d'un pluralisme inutile.

L'ordre des conférenciers, lors des trois sessions semiplénières, a ensuite suivi la logique du micro au macro - c'est-à-dire des approches individualistes vers des démarches plus institutionnaliste 4 . Marco Janssen (mathématicien, Arizona State University, États-Unis) a introduit le thème de la première session, l'interface entre les systèmes sociaux et écologiques, par son allocution sur la convergence entre les institutions et les dynamiques

\footnotetext{
4 En termes schématiques, les approches «individualistes » cherchent des explications aux phénomènes sociaux dans les caractéristiques et les comportements des individus, tandis qu'une démarche institutionnaliste met l'accent sur le jeu des institutions, telles que les normes collectives, les habitudes des individus et les marchés.
} 
des écosystèmes5. Wander Jager (professeur de marketing, Université de Groningue, Pays-Bas), s'est ensuite focalisé sur la notion de complexité sociale et sur sa pertinence pour l'économie écologique.

Les approches économiques du changement climatique ont constitué le thème de la deuxième session. Lučka Kajfež Bogataj (climatologue, Université de Ljubljana) a préconisé l'internalisation des coûts environnementaux externes, tandis que Richard B. Howarth (économiste, Darthmouth College, États-Unis) - qui est le rédacteur en chef de la revue Ecological Economics depuis janvier 2008 - a fourni une critique élaborée des approches néoclassiques du risque dans les modèles climatiques.

Enfin, la troisième session a adopté la perspective la plus large : l'interaction entre la nature et la société au niveau systémique. La tâche attribuée à Susan Baker (professeur en sciences sociales, Cardiff University, Royaume-Uni), était ambitieuse : donner un aperçu global de la gouvernance du développement durable, d'un point de vue des sciences politiques. Zbigniew W. Kundzewicz (hydrologue, Académie des sciences de Pologne) a fourni des pistes pour comprendre le paradoxe selon lequel le «scepticisme climatique» dans les pays de l'ancien bloc soviétique semble augmenter au fur et à mesure que s'accumulent les preuves de l'origine anthropique des changements climatiques. Enfin, Frank Biermann (professeur de sciences politiques, Université libre d'Amsterdam, Pays-Bas) a présenté, lors de la séance de clôture, la nouvelle phase du programme de recherche Earth System Governanc] dont il est président du comité scientifique.

\section{Les participants}

En raison du lieu de la conférence, il n'est guère surprenant que la participation des chercheurs d'Europe centrale et orientale ait été relativement forte. Cependant, malgré les efforts de l'ESEE pour s'élargir à l'Est, l'économie écologique peine à gagner en crédibilité dans ces pays. L'absence d'une tradition des sciences sociales critiques, ainsi que la peur d'être marginalisé sur le marché du travail expliquent sans doute en partie cette inertie.

\footnotetext{
5 C'est l'idée selon laquelle les institutions et les dispositifs de gestion d'un écosystème doivent être revêtus des mêmes caractéristiques structurelles et fonctionnelles que celui-ci.

${ }^{6}$ En particulier, l'économie néoclassique tend à traiter l'incertitude (par exemple, face aux impacts incertains, mais potentiellement irréversibles dus au changement climatique) comme si elle était réductible à un risque. Cette approche permet d'intégrer le risque dans les calculs de probabilité et de définir un «optimum » en comparant les différents risques sur une échelle commune. En désaccord avec cette approche qu'elle juge réductrice, l'économie écologique prône l'adoption du principe de précaution face aux incertitudes «fortes ».

${ }_{7}$ Cf. http://www.earthsystemgovernance.org.
}

Un aspect positif, en revanche, était la forte participation des étudiants et des jeunes chercheurs, tendance qui ne cesse de se renforcer, grâce notamment à la multiplication des écoles d'été et des cursus universitaires en économie écologique.

Comme si souvent dans les colloques internationaux anglophones, les chercheurs français ont été peu nombreux. Il est à noter cependant, que leur origine institutionnelle s'est diversifiée (l'Université de Versailles SaintQuentin-en-Yvelines ne détient plus le quasi-monopole de la discipline dans ce pays) et que la qualité des contributions françaises a sans doute compensé leur faible nombre. En témoigne le fait que l'un des deux prix de la meilleure intervention étudiante a été attribué à une Française, Catherine Jolibert (Université autonome de Barcelone), pour sa communication «Need assessment in multi-level governance: a tool for environmental conflict? ».

\section{Les thèmes du colloque}

Même si l'on ne peut pas tirer de conclusions définitives sur l'état d'une discipline à partir d'un seul colloque, les thèmes abordés dans les nombreuses sessions, ainsi que les discussions qui se sont poursuivies à l'extérieur permettent de formuler un certain nombre d'observations.

Que le changement climatique ait occupé une place primordiale parmi les thèmes du colloque paraît logique et justifié. L'économie écologique se caractérise, en effet, par son souci de proposer des solutions pertinentes aux problèmes du «monde réel ». Par ailleurs, l'ESEE fait preuve d'une maturité parfois étonnante, comme en témoignent tout à la fois la constance des thèmes centraux abordés par la discipline et les évolutions progressives des débats méthodologiques. Plusieurs points méritent d'être mentionnés à ce propos.

Tout d'abord, le nombre des contributions purement néoclassiques diminue régulièrement depuis la fondation de la Société européenne en 1996, ce qui atteste d'une certaine maturation de la discipline. De même, si l'évaluation environnementale reste un thème de recherche important de l'économie écologique - avec ses critiques à l'égard de l'analyse coûts-bénéfices et des méthodes agrégatives -, le développement des approches concrètes et l'opérationnalisation du principe d'incommensurabilitê - désormais accepté comme un principe

\footnotetext{
${ }^{8}$ En sciences économiques, l'incommensurabilité peut être définie comme le principe selon lequel la comparaison et le choix entre des valeurs qui sont de nature fondamentalement différente (par exemple, la valeur d'un écosystème et la valeur de la production à partir des ressources naturelles de ce même écosystème) ne peuvent pas se faire en ayant recours à une mesure de valeur commune comme base de comparaison. L'absence d'une unité de mesure commune n'empêche pas pour autant le développement d'autres méthodes de comparaison : les méthodes d'évaluation constituent ainsi l'un des axes de recherche au cœur de l'économie écologique.
} 
fondamental dans l'économie écologique - constituent aujourd'hui le cœur des réflexions. Par ailleurs, ces travaux méthodologiques cherchent de plus en plus systématiquement à placer l'évaluation dans un contexte de gouvernance plus large. Un nouveau thème est ainsi désormais «à la mode», notamment chez les doctorants : l'approche «paiements pour services écosystémiques ».

Un autre thème constant de l'économie écologique, l'analyse du «métabolisme sociétal » a également connu des évolutions. Les études mobilisant l'empreinte écologique ou l'intensité matérielle des biens et services, désormais conventionnelles, sont de plus en plus complétées par l'évaluation de l'appropriation humaine de la production primaire nette (Human Appropriation of Net Primary Production [HANPP] $)^{9}$. Une originalite de cette approche est son ambition d'intégrer, dans l'analyse des pressions sur la biodiversité occasionnées par les activités économiques, un examen des questions d'équité et de distribution.

En ce qui concerne les recherches sur les liens entre croissance des revenus, environnement et bien-être, on note une baisse d'intérêt pour la notion de courbe de Kuznets environnementale10, et, en parallèle, une augmentation des études sur les mesures alternatives du bien-être. Nombre de présentations dans le colloque ont adopté l'approche par les capacités (capabilities en anglais), développée par Amartya Sen, l'un des membres de la commission Stiglitz-Sen-Fitoussi, née d'une demande de Nicolas Sarkozy en 2008 d'élaborer de nouveaux indicateurs de progrès social plus pertinents que le PIB 11 .

Par ailleurs, les courants de l'économie cognitive et de l'économie comportementale semblent vivre une renaissance au sein de l'ESEE. Mais cette société n'aura de réels apports dans ces domaines qu'à condition d'éviter une approche individualiste excessive et de s'appuyer, en revanche, sur la forte tradition institutionnaliste de la communauté. La mise en perspective de macrogouvernance des dispositifs délibératifs et participatifs

${ }^{9}$ L'HANPP cherche à estimer l'intensité de l'utilisation des écosystèmes par la société dans des territoires spécifiques. Contrairement à une mesure similaire, l'empreinte écologique, l'HANPP ne calcule pas la pression totale qu'exerce la consommation de la société sur la biosphère.

10 En anglais, «Environmental Kuznets Curve» (EKC). Cette courbe illustre les liens entre les pressions sur l'environnement et le revenu par habitant dans une société donnée.

11 Le rapport de cette commission est paru en septembre 2009. Il est disponible sur le site Internet http://www. ladocumentationfrancaise.fr/rapports-publics/094000427

${ }^{12}$ Ces courants de pensée s'inspirent des théories de l'économie évolutive et institutionnelle, mais aussi de la psychologie. Par opposition à l'hypothèse de l'économie néoclassique de l'acteur rationnel - Homo æconomicus -, ils adoptent la notion de l'acteur à rationalité limitée et ils étudient l'interaction des aspects sociaux, émotionnels et cognitifs, afin d'expliquer le comportement des agents économiques. développés à l'échelle locale figure sans doute parmi les candidats les plus prometteurs pour de tels apports. Une autre voie de recherches dans cette même lignée est le développement d'outils d'analyse des modes de consommation individuelle, à travers une approche intégrant deux traditions théoriques : 1'économie comportementale d'un côté, et les transitions dans les systèmes sociotechniques 13 , de l'autre.

\section{La crise financière : une fenêtre d'opportunité politique ou théorique?}

De nombreux participants ont mentionné l'opportunité que la crise économique et financière actuelle fournit à l'économie écologique pour se faire entendre et pour gagner en crédibilité politique. Pourtant, il n'est pas sûr que la discipline soit prête à saisir cette occasion : possède-t-elle les outils nécessaires pour analyser la situation macroéconomique actuelle et pour ainsi formuler des recommandations politiques pertinentes? Le colloque de Ljubljana a sans doute marqué le début d'une réflexion sérieuse sur ce que serait une "macroéconomie écologique ». Jusqu'à présent, l'économie écologique s'est surtout définie en des termes microéconomiques qui s'opposaient aux préceptes de la microéconomie environnementale néoclassique. Mais, bien qu'il soit nécessaire de continuer à développer des alternatives à l'internalisation monétaire des coûts externes et des méthodes d'évaluation multicritères et délibératives, cela ne dispense pas d'une réflexion macroéconomique. On peut donc dire que la crise actuelle a sans doute rendu service à l'économie écologique en pointant du doigt ses lacunes en macroéconomie et en lui permettant d'initier un début de réflexion sur le sujet. Parmi les pistes à explorer, on retiendra une collaboration plus étroite avec les post-keynésiens ainsi qu'un travail plus rigoureux et scientifique sur les théories de la décroissance jusqu'ici dominées par des approches idéologiques et militantes.

\section{Les frontières de l'économie écologique}

Un certain nombre de questions pertinentes concernant les frontières de la discipline ont également été abordées. Un premier enjeu - sujet de longue date dans la communauté - porte sur les dissensions entre les adeptes du militantisme et ceux d'une recherche "détachée».

\footnotetext{
${ }^{13}$ Dans ce courant de recherche, l'objet de l'analyse réside dans la complexe interaction et dans la coévolution des éléments - technologie, politique, environnement, réglementation et culture-qui constituent les systèmes dits «sociotechniques ». L'accent est mis sur la complexité et l'adaptabilité des systèmes, ainsi que sur l'apprentissage, l'expérimentation et l'itération, souvent dans une ambition de promouvoir la gestion plus « durable » de ces systèmes.
} 
D'un côté, les pragmatistes écologistes sont prêts à se servir de tout outil pour faire avancer la cause environnementale. Cette approche, traditionnellement forte au sein de la Société américaine de l'économie écologique, s'oppose à celle qui voit avant tout l'économie écologique comme une discipline scientifique. Le colloque de Ljubljana n'a pas échappé à cette tension : une motion a été déposée pour que l'ESEE se déclare en faveur d'une économie de l'état stationnaire. Son rejet, après débat, a marqué, cette fois-ci, la défaite du courant militant, sans pour autant signifier que la question soit close définitivement.

Une autre question pertinente concerne la place de l'économie écologique au sein des sciences économiques. Certains voient la discipline comme un moyen de réformer l'économie néoclassique de l'intérieur et s'opposent à d'autres qui jugent une telle ambition naïve et vouée à l'échec, voire dangereuse. Pour ces derniers, la stratégie des premiers ne mènerait qu'à l'absorption de l'économie écologique par le courant dominant, qui ne subirait que des modifications mineures. Ils soutiennent, par ailleurs, qu'en tentant cette réforme de l'intérieur, l'économie écologique a déjà perdu un temps précieux qu'elle aurait pu employer pour des travaux méthodologiques, notamment dans le domaine de la macroéconomie.

Reste enfin l'interface entre l'économie écologique et les autres sciences sociales, notamment les sciences politiques. Bien que l'ESEE ait intégré, depuis sa naissance, un fort contingent de socioéconomistes et de chercheurs d'autres disciplines des sciences sociales et politiques, les relations à mettre en place avec ces domaines sont complexes. D'un côté, une économie écologique «pure», vivant en vase clos, n'est guère possible ni souhaitable. De l'autre, l'intérêt de chaque rapprochement avec l'une de ces sciences sociales et politiques doit être évalué en fonction des plus-values que peuvent s'apporter réciproquement chacune de ces disciplines et l'économie écologique.

Enfin, l'économie écologique doit également définir ses rapports avec de nombreuses autres jeunes communautés de la recherche interdisciplinaire. Ainsi, l'analyse des systèmes complexes s'est développée au sein de l'économie écologique, en partie grâce à la collaboration avec des chercheurs travaillant sur les transitions dans les systèmes sociotechniques. Un autre interlocuteur naturel est le courant Interpretive Policy Analysis (IPA), encore en quête de son identité ${ }^{14}$. Cependant, bien que l'ESEE ait déclaré son intention de renforcer les collaborations avec ses «sœurs », la question de la forme qu'elles prendront persiste, ainsi que la question des frontières : jusqu'où peut-on aller dans le pluralisme sans perdre son identité?

\section{L'avenir}

Dans les deux ans à venir, deux événements importants pour la communauté de l'économie écologique méritent d'être mentionnés. Le premier verra le retour en Europe, après plus de dix ans d'absence, de la conférence de l'International Society for Ecological Economics $(\text { ISEE })^{15}$. Entre 600 et 800 participants sont attendus, du 22 au 25 août 2010, à Brême et à Oldenbourg en Allemagne ${ }^{16}$. Le second, la prochaine conférence de l'ESEE, aura lieu à Istanbul du 15 au 18 juin 2011.

\footnotetext{
${ }^{14}$ L'IPA est un courant d'analyse politique, critique à l'égard des approches positivistes plus traditionnelles, qui adhère au pluralisme méthodologique et qui souligne l'importance des aspects tels que la signification, le langage et le discours, le contexte historique et social, ainsi que la subjectivité humaine dans l'analyse des phénomènes politiques. Pour plus d'informations sur ce courant et la conférence IPA de juin 2010 à Grenoble, $c f$. http://www.ipa2010-grenoble.fr.

15 En français, Société internationale pour l'économie écologique. Cf. http://www.ecoeco.org

16 Pour plus d'informations sur cette conférence, consultez le site Internet/http://wwsw.isee2010.org
} 